\title{
Influence of X-ray radiation on the non-LTE wind models of O-type stars
}

\section{Jiří Krtička}

Institute for Theoretical Physics and Astrophysics, Faculty of Sciences, Masaryk University, Kotlarska 2, CZ - 61137 Brno, Czech Republic

email: krticka@physics.muni.cz

\begin{abstract}
We study the influence of shock X-ray emission on the structure of non-LTE wind models of O-type stars.
\end{abstract}

Keywords. stars: early-type, stars: atmospheres, stars: winds, outflows

We study the influence of shock X-ray emission on the structure of non-LTE wind models of O-type stars. For this purpose we use our own non-LTE wind code with an artificial X-ray source to understand the influence of X-rays on the wind ionization structure and, consequently, on the basic wind parameters of O-type stars (mass-loss rate and the terminal velocity). The inclusion of X-ray sources into wind models enables to obtain wind ionization structure which is more consistent with the observed one. However, the presence of strong X-ray radiation does not significantly influence the mass-loss rate, but it may influence the terminal velocity for cooler O-type stars. Wind models with constant X-ray filling factor are able to roughly explain the observed relation between the X-ray luminosity and the total luminosity. Star exhibiting so-called 'weak wind problem' produce X-rays apparently more efficiently than the 'normal' stars. We discuss the implications of wind models with X-ray emission on the current problems of hot star wind research, namely the discrepancy between wind properties derived from observation and theoretical wind models. (Poster Presentation.) 